\title{
NUTRITION AND HEART DISEASE: A REVIEW
}

\author{
Emmanuel, O. B. B \\ Department of Hospitality and \\ Tourism Management, \\ Federal University Wukari, \\ Nigeria
}

\author{
Abdussalaam, R. O \\ Department of Hospitality and \\ Tourism Management, \\ Federal University Wukari, \\ Nigeria
}

\author{
Ali, J. E \\ Department of Hospitality and \\ Tourism Management, \\ Federal University Wukari, \\ Nigeria
}

\begin{abstract}
Heart disease is one of several cardiovascular diseases, which are disorders of the heart and blood vessel system. Heart disease describes a range of conditions that affect the heart. The narrowing or blockage of the blood vessels that supply blood to the heart itself (coronary arteries) is the major cause of heart disease and it happens slowly over time. The major likely cause of coronary arteries blockage is the dietary pattern of individuals. Heart disease tendencies can be reduced by preventing or controlling behaviours and conditions known to increase its risk (risk factors) which are classified into two: those that can be changed and those that cannot be changed. Many forms of heart disease can be prevented or treated with healthy lifestyle choices. Different diets have been shown to reverse heart disease. Some of these diets are vegan and very low in fat, some include animal protein and have more good fats, all the diets are high in plants and fibre (vegetables, fruits, whole grains), all severely restrict processed foods like white flour, sugar, and so on. Some of the diets go alongside with supplements, exercise, stress management and social support.
\end{abstract}

Keywords - Heart disease, nutrition, heart healthy diet, reversal diet

\section{INTRODUCTION}

Heart disease is the leading cause of death in both men and women (Valencia and Kristeen, 2016) and statistics showed that it is the major cause of death globally (Mozaffarian et al., 2015). Heart disease is one of several cardiovascular diseases, which are disorders of the heart and blood vessel system (Sandmaier, 2005). Heart disease describes a range of conditions that affects the heart's structure and function (American Heart Association, 2002; Christian, 2016; Valencia and Kristeen, 2016). All heart diseases are cardiovascular diseases, but not all cardiovascular diseases are heart disease (National Heart, Lung, and Blood Institute, 2017).

Coronary heart disease is the deadliest of all heart diseases and it is the most common form (Valencia and Kristeen, 2016). Coronary heart disease is the most common type of heart disease, killing nearly 380,000 people annually (Benjamin, 2016). Deaths caused by heart disease are projected to increase sharply between 2010 and 2030, and the population of heart disease survivors is expected to grow at a much faster rate than the U.S. population as a whole (AHA, 2002). The average age of death from coronary artery disease in the developed world is around 80 while it is around 68 in the developing world (Bridget, 2010).

Cholesterol level has a tendency of increasing in individuals who eat foods that are rich in saturated fats and cholesterol (AHA, 2008). Diets rich in animal protein having higher amounts of fatty acids are converted to various lipoproteins in the liver leading to increased deposition of adipose tissue, facilitating formation of atheromatous plaques inside the arteries (Walker et al., 2005). As a result, there is narrowing of the arterial wall leading to increased risk of hypertension, stroke and coronary arterial diseases (Sauvaget et al., 2004; Walker et al., 2005). A low-fat diet has been reported to decrease low density lipoprotein cholesterol (LDLc) levels (Pelkman et al., 2004). Very high carbohydrate intake is accompanied by a reduction in high density lipoprotein cholesterol (HDL-c) and a rise in triglyceride (Miller et al., 2011). Elevated triglyceride and decreased high density lipoprotein cholesterol (HDL-c) serum levels are both risk factors for developing coronary heart disease (Ma et al., 2006). Consumption of fruits and vegetable based diets, rich in fiber, folic acid, antioxidants, and phytochemicals are associated with lower serum cholesterol (Djousse et al., 2004; Craig, 2009). Whole grain consumption has been shown to improve lipid profiles (Borneo and Leon, 2012). Therefore this paper highlights the significance of nutrition in the management of heart disease.

\section{HEART DISEASE}

Heart disease is the same as cardiac disease but not the same as cardiovascular disease (Christian, 2016). Cardiovascular disease refers to disorders of the blood vessels and heart, while heart disease refers to disease conditions that affect just the heart (Christian, 2016).

The most common type of heart disease is coronary heart disease and when people talk about "heart disease" they often mean coronary heart disease (Valencia and Kristeen, 2016). Coronary heart disease is often simply called heart disease (AHA, 2002; Sandmaier, 2005).

\section{A. Types and symptoms of heart disease}

Diseases under heart disease includes coronary artery disease, heart rhythm problems (arrhythmias), and heart defects one is 


\section{International Journal of Engineering Applied Sciences and Technology, 2019 \\ Vol. 4, Issue 3, ISSN No. 2455-2143, Pages 391-399 \\ Published Online July 2019 in IJEAST (http://www.ijeast.com)}

born with (congenital heart defects), among others (Christian, 2016).

Heart disease is often called a "silent killer" as it may not be diagnosed until there are signs of a heart attack or heart failure (Valencia and Kristeen, 2016). Symptoms of heart disease vary depending on gender and the specific condition (Valencia and Kristeen, 2016).

\section{B. Causes of heart disease}

The narrowing or blockage of the coronary arteries is the major cause of heart disease and it happens slowly over time (Sandmaier, 2005). The major likely cause of coronary arteries blockage is the dietary pattern of individuals (Hu, 2003). Heart disease occurs when the coronary arteries, which supply blood to the heart muscle, become hardened and narrowed due to build up of plaque on the arteries inner walls (Sandmaier, 2005; Valencia and Kristeen, 2016). Plaque is the accumulation of cholesterol, fat, and other substances (Sandmaier, 2005). As plaque continues to build up in the arteries, blood flow to the heart is reduced leading to heart disease (Sandmaier, 2005; Valencia and Kristeen, 2016).

\section{Risk factors of heart disease}

The eating habits and other lifestyle factors play a large role in determining the risk of heart disease and may prevent or even reverse this condition (Esselstyn, 2016). Heart disease tendency can be reduced by preventing or controlling behaviours and conditions known to increase its risk (risk factors) (Sandmaire, 2015). Risk factors associated with heart disease are classified as modifiable factors and non-modifiable (those that can be changed and those that cannot be changed) (Sandmaire, 2015). Non-modifiable risk factors are beyond the control of an individual and include age, sex, family history of premature cardiovascular events, and race (Libby, 2005). Modifiable risk factors are those that are as a result of circumstances and are under the control of an individual and this includes hypertension, diabetes mellitus (DM), dyslipidaemia, tobacco smoking, stress, poor hygiene, poor diet, obesity, and sedentary life style (Libby, 2005). However, it is important to note that some risk factors such as diabetes mellitus fall in both categories as their aetiology is affected by both behaviour and genetic (Morgan, 2016).

\section{NUTRITION AND HEART DISEASE MANAGEMENT}

A healthy nutrition lifestyle can go a long way to solving heart disease problem. The advanced countries have easy access to abundant high fat food and ironically, it is this "rich diet" that produces atherosclerosis while in poorer nations, many people manage to survive on a primarily plant based diet, which is far healthier, especially in terms of heart disease (Esselstyn, 2016).

What is eaten can help keep the heart beating strong or lead to overweight, high blood pressure, and high blood cholesterol which are three key factors that increase the risk of developing heart disease (Healthwise, 2015). According to Esselstyn (1999), stenting and bypass surgeries may only be a solution for a minority of patients, while a transition to a plant based diet free of cholesterol proves to be a more effective treatment for heart disease. At least $80 \%$ of premature deaths from heart disease and stroke could be avoided through healthy diet, regular physical activity and avoiding tobacco smoke (WHO, 2017).

\section{A. Heart healthy diet}

According to the National Heart, Lung, and Blood Institute (2003), cooking up heart healthy diet requires no secret ingredients but it is simply making dishes that are lower in saturated fat, cholesterol, and total fat, and reduced in sodium. An extra plus is that these dishes have fewer calories than those dishes that are higher in fat (N.I.H, 2003; Esselstyn, 1999).

No single food can make one magically healthy, so the overall dietary pattern is more important than specific foods (Pretorius and Mpe, 2016; Lawrence et al., 2017). Instead of fried, processed food, packaged meals, and sugary snacks, a heart healthy diet is built around "real," natural food fresh from the ground, ocean, or farm (Pretorius and Mpe, 2016; Lawrence et al., 2017). A heart healthy diet has lots of vegetables, fruits, nuts, dried beans, and whole grains, and is low in sodium (N.I.H, 2003). It limits foods that are high in saturated fats, trans-fats, and cholesterol (N.I.H, 2003). Trans-fatty acids increase coronary heart disease risk compared with other macronutrients (Brouwer et al., 2010). The following are the dietary guidelines to manage and prevent heart disease.

\section{Decrease cholesterol intake}

Cholesterol should be limited to less than 300 milligrams daily for healthy individuals and less than 200 milligrams daily for those with heart disease, diabetes, or high low density lipoprotein (LDL) cholesterol (Bellows and Moore, 2013). When cutting down on cholesterol, choosing lean cuts of meat is not enough because the cholesterol is mainly in the lean portion (Physician Committee, 2018). Chicken contains as much cholesterol as beef (Scherr and Zidenberg-Cherr, 2016) and shellfish are also very high in cholesterol and therefore all animal products should be avoided for this reason (Physician Committee, 2018). No food from plants contains cholesterol, since plants do not have a liver to produce it (Sandi, 2018). Every $100 \mathrm{mg}$ of cholesterol in the daily diet adds roughly five points to the level of cholesterol in the body, although this varies from person to person and every one percent in reduction of one's cholesterol level reduces the risk of heart disease by two percent (Physician Committee, 2018).

2. Decrease fat intake (Saturated fats and trans fats) Fat intake should be decreased especially saturated fat because it causes the liver to produce more cholesterol however unsaturated fats do not have this effect (Scherr and ZidenbergCherr, 2016). Healthy individuals without heart disease should limit saturated fat to less than $10 \%$ of total daily 


\section{International Journal of Engineering Applied Sciences and Technology, 2019 Vol. 4, Issue 3, ISSN No. 2455-2143, Pages 391-399 \\ Published Online July 2019 in IJEAST (http://www.ijeast.com)}

calories, while those with heart disease, diabetes, or high low density lipoprotein (LDL) cholesterol should limit intake of saturated fat to less than $7 \%$ of total daily calories and all individuals should limit trans fatty acid to less than $1 \%$ of the total daily calories (National Heart foundation of Australia and new Zealand, 2012; Bellows and Moore, 2013; Pretorius and Mpe, 2016). Saturated fats are solid at room temperature, whereas unsaturated fats are liquid (Kathleen and Forrest, 2009). Beef, chicken, and most other animal products contain substantial amounts of saturated fat and this is another good reason to avoid these foods (Atli, 2015). Foods high in fat (60$100 \%$ fat) are cheeses, butter, margarine, and oils of all types while grains, beans, vegetables, and fruits have less than 10 percent of their calories coming from fat (Physician Committee, 2018). Tropical oils (palm oil, palm kernel oil, and coconut oil) are also high in saturated fats (Kathleen and Forrest, 2009; Pretorius and Mpe, 2016). Many packaged foods contain hydrogenated oils that are both high in saturated and trans-fat and are chemically hardened to make them solid at room temperature to increase their shelf life (Physician Committee, 2018). While liquid vegetable oils are much better than animal fats and tropical oils, all fats and oils are natural mixtures of saturated and unsaturated fats and therefore, they are all not good for the coronary arteries and consumption should be kept to a minimum (Kathleen and Forrest, 2009; Physician Committee, 2018). Eating foods rich in monounsaturated and polyunsaturated fat can improve blood cholesterol levels and lower the risk of heart disease (Lawrence et al., 2017). Sources of healthy fats include olive oil, canola oil, peanut oil, avocados, soy, seeds, nuts, and nut butters (Lawrence et al., 2017). Decreasing fat intake can be achieved be eating mainly plant-based foods (e.g. fruits, vegetables, pulses and a wide selection of wholegrain foods), moderate amounts of reduced, low or no fat dairy products, moderate amounts of lean unprocessed meats, poultry and fish and also avoiding fried foods, fast food and processed foods (Bellows and Moore, 2013). Also, limit intake of tropical oils, dairy, and red meat (Physician Committee, 2018). Enjoy dairy in moderation and vary the protein sources in the diet, opting for fish, skinless chicken, eggs, and vegetarian sources of protein (Pretorius and Mpe, 2016). Avoid deep fried foods, packaged foods labelled as "hydrogenated" or "partially hydrogenated" oils (Lawrence et al., 2017).

3. Foods containing omega 3 and omega 6 fatty acids Oily fish such as mackerel, sardines, whitefish, tuna and salmon contains omega 3 fatty acids (Andrea, 2018). This type of fat has been shown to decrease triglycerides and increase high density lipoprotein (HDL) cholesterol levels, improves blood vessel elasticity and thins the blood, making it less likely to clot and block blood (Better Health Channel, 2012). The consumption of two servings a week of fish high in eicosapentaenoic acid (EPA) and docosahexaenoic acid (DHA) is associated with a reduced risk of both sudden death and death from coronary heart disease in adults (Pretorius and
Mpe, 2016). Some vegetables oils such as corn, soy and safflower, contains omega 6 fatty acids, and canola and olive oil contains omega 3 fatty acids (Better Health Channel, 2012). All of these can help to lower low density lipoprotein (LDL) cholesterol when used instead of saturated fats such as butter (Physician Committee, 2018).

\section{4. $\quad$ Foods of plant origin}

The amount and variety of plant foods consumed should be increased. A diet based on plant foods such as grains, beans, vegetables, and fruits are the best way to keep saturated fat intake low and to avoid cholesterol completely (Bellows and Moore, 2013). Eating only plant based foods (a diet free of all animal products) like vegetarians yields the lowest risk of heart disease (Lawrence et al., 2017). A study reported that vegetarians had a much lower risk of dying from heart disease than non vegetarians (Craig and Mangels, 2009). A high-fat fast food meal usually has 42 grams of fat, while a typical healthful meal would have around 1.3 grams of fat (Jakulj et al., 2007). An additional benefit of eating a plant based diet like the vegetarians is that there is reduced iron storage in vegetarians and study shows that there is a strong link between iron and heart disease and hypertension (Physician Committee, 2018).

\section{Vegetables and fruits}

Fruits and vegetables are low fat and do not contain cholesterol (Kathleen and Forrest, 2009). The antioxidants in fruits and vegetables offer protection against heart disease and they also contain various phytonutrients that promote heart health (Bellows and Moore, 2013). They are also important sources of folate, which helps lower the blood levels of the amino acid homocysteine, which appears to be linked to an increased risk of heart disease (Better Health Channel, 2012). Eating at least five servings of fruit and vegetables a day, and limiting salt intake to less than one teaspoon daily, also helps to prevent heart attacks and strokes (Lawrence et al., 2017). Another study reported that consuming 5-10 servings of fruits and vegetables daily also reduces coronary heart disease (CHD), stroke, and blood pressure (Pretorius and Mpe, 2016; Physician Committee, 2018). Leafy green vegetables are high in vitamin $\mathrm{K}$ which helps protect the arteries and promote proper blood clotting (Maresz, 2015) and it also contains nitrates, which have been shown to reduce blood pressure, decrease arterial stiffness and improve the function of cells lining the blood vessels (Vikas et al., 2014).

\section{Legumes and Soy protein}

Soy protein has been shown to lower low density lipoprotein (LDL) cholesterol levels, especially if blood cholesterol levels are high (Better Health Channel, 2012). It has been reported that replacing animal protein with soy protein reduces blood cholesterol levels (Lichtenstein et al., 2006) even when the total amount of fat and saturated fat in the diet remains the same (Physician Committee, 2018).

\section{Foods containing fiber}




\section{International Journal of Engineering Applied Sciences and Technology, 2019 Vol. 4, Issue 3, ISSN No. 2455-2143, Pages 391-399 \\ Published Online July 2019 in IJEAST (http://www.ijeast.com)}

A diet high in fiber can lower low density lipoprotein (LDL) cholesterol and provide nutrients that help protect against heart disease (Lawrence et al., 2017). Fiber moves fat through the digestive system quicker so less of it is absorbed (Lawrence et al., 2017; Rachael, 2018). Soluble fiber slows the absorption of some food components, such as cholesterol, and reduces the amount of cholesterol the liver produces (Lawrence et al., 2017). Soluble fiber traps cholesterol by forming a gel-like substance and it binds to cholesterol, it works to prevent cholesterol absorption and promote cholesterol excretion while insoluble fiber provides bulk to stool (Andrea, 2018). Every 10 grams of fiber per day reduces the risk of dying by 10 percent (Chuang et al., 2012). There is no fiber in any animal product (Kathleen and Forrest, 2009; Sandi, 2018). Insoluble fiber is found in whole grains, wheat cereals, and vegetables such as carrots, celery, and tomatoes while soluble fiber sources include barley, oatmeal, beans, nuts, vegetables and fruits such as apples, berries, citrus fruits, grapes prunes, strawberries, carrots, eggplant, okra, and peas (Lawrence et al., 2017; Andrea, 2018).

\section{Nuts and seeds}

Nuts and seeds are high in kilojoules (Better Health Channel, 2012). Despite their high energy density, nuts do not contribute to weight gain, changes in waist circumference, or obesity, perhaps due to their satiating effects and increased fecal energy losses (Smith et al., 2015). They are good sources of heart friendly fats and fiber and higher intake of nuts can protect the heart through lowering total cholesterol and low density lipoprotein (LDL) cholesterol levels (Andrea, 2018). Studies have also found that regularly eating nuts such as walnuts is associated with a lower risk of heart disease ( $\mathrm{Li}$ et al., 2009; Aune, 2016). It has been found that adding seeds to the diet can improve many heart disease risk factors, including inflammation, blood pressure, cholesterol and triglycerides (Rachael, 2018).

\section{Foods enriched with plant sterols and stanols}

A daily intake of 2-3g of phytosterols/stanols lowers low density lipoprotein (LDL) cholesterol levels by approximately $10 \%$ in healthy people and in those with high cholesterol and diabetes (Better Health Channel, 2012). This intake can be achieved by the consumption of two to three serves of phytosterol enriched foods like reduced fat yoghurts, milk and breakfast cereals, alcohol, fruits and vegetables (National Heart foundation of Australia and New Zealand, 2012).

\section{Foods containing vitamin $\mathbf{E}$}

Some studies indicate that vitamin $\mathrm{E}$ acts as an antioxidant, helping to protect against low density lipoprotein (LDL) cholesterol (Better Health Channel, 2012; Kathleen and Forrest, 2009). The risk of heart disease is significantly lowered in populations that consume the highest levels of Vitamin E and taking Vitamin E supplements (100-400 IU per day) may decrease the risk of getting heart disease (Kathleen and Forrest, 2009). Good sources of vitamin E include avocados, nuts, dark green vegetables, vegetable oils and wholegrain products (Better Health Channel, 2012).

\section{Garlic, turmeric and onion}

There is a compound in fresh garlic called allicin and this compound has been found in some studies to lower blood cholesterol (Better Health Channel, 2012; Ried, 2016). Allicin is a sulfur compound and it is responsible for garlic's distinctive smell and taste (Stephen, 2018a). Allicin helps to keep the blood thin and flowing smoothly, as well as regulate blood pressure and maintain healthy cholesterol levels (modest reductions in total serum and low density lipoprotein cholesterol levels) (Ried, 2016; Stephen, 2018a). It has been reported that the active compound in turmeric called curcumin blocks a wide range of biochemical reactions involved in cardiac hypertrophy (enlargement of the heart chambers) and it helps support healthy blood pressure (Stephen, 2018b). According to Channi-Tiwary (2018), raw onion lowers the production of low density lipoprotein (LDL) and keeps the heart healthy.

\section{Green tea}

Some evidence suggests that the antioxidants in green tea can help prevent the buildup of fatty deposits in the arteries (Better Health Channel, 2012). The antioxidants may also act as an anti-blood clotting agent and improve blood vessel dilation to allow increased blood flow (Better Health Channel, 2012; Rachael, 2018).

\section{Alcohol}

It is thought that a moderate intake of alcohol may have some potential health benefits. For example, some types of alcohol (such as red wine) may contain protective factors like antioxidants, although this is still being researched (Better Health Channel, 2012). Alcohol also increases the high density lipoprotein (HDL) cholesterol and this helps clear cholesterol from the body (Pretorius and Mpe, 2016). However, a high intake of alcohol increases blood pressure and also tends to increase triglycerides in the blood, increasing the risk of heart disease (Sandmaier, 2005). Current guidelines for alcohol intake recommends not more than two standard drinks per day for men and one for women to reduce the risk of harm related to alcohol (National Heart foundation of Australia and new Zealand, 2012).

\section{Packaged and processed foods}

Avoid packaged and processed foods especially those high in sodium and sugar (Lawrence et al., 2017). Processed meat such as bacon, sausage, and salami, and fried chicken. Minimize intake of beverages and foods with added sugar (Scherr and Zidenberg-Cherr, 2016). The body does not need any added sugar because it gets all it needs from the sugar that naturally occurs in food (Lawrence et al., 2017). Sugary food and refined carbohydrates increases the number of empty calories that are bad for the heart and also for the waistline (Pretorius and Mpe, 2016). Reduce intake of refined sources of carbohydrates with higher 


\section{International Journal of Engineering Applied Sciences and Technology, 2019 Vol. 4, Issue 3, ISSN No. 2455-2143, Pages 391-399 \\ Published Online July 2019 in IJEAST (http://www.ijeast.com)}

glycaemic indices (Better Health Channel, 2012). Instead of taking sugary soft drinks, white bread, pasta and processed foods like pizza it is better to take unrefined whole grains like whole wheat or multigrain bread, brown rice, barley, bran cereal, oatmeal, and non starchy vegetables (Pretorius and Mpe, 2016).

\section{Limit sodium intake}

Intake of sodium rich foods like processed meats, some frozen and canned products can contribute to high blood pressure (Andrea, 2018). Avoid cooking salty foods and also adding salt at the table (Kathleen and Forrest, 2009; Lawrence et al., 2017). Sodium intake can be reduced by reducing the intake of canned or processed foods, salty snacks, salty takeaway foods, choosing foods processed without salt, foods labelled 'no added salt' or 'low salt', or foods labelled 'reduced salt' and eating fresh foods, unsalted meats, and homemade food (Pretorius and Mpe, 2016; Lawrence et al., 2017).

Always

check the sodium content of foods and choose the

lowest sodium products (Lawrence et al., 2017). Not more than $2,300 \mathrm{mg}$ of sodium is recommended daily for adults (Bellows and Moore, 2013).

16. Use heart healthy cooking methods.

Foods can be baked, broiled, roasted, steamed, poached, lightly stir fry, or sautéed (Lawrence et al., 2017).

\section{B. Heart disease reversal diets}

Different diets have been shown to reverse heart disease. Some are vegan and very low fat, some include animal protein and have more good fats, some include supplements, all are high in plants and fiber: vegetables, fruits, whole grains, all severely restrict processed foods: white flour, sugar, pasta (Buckley, 2017). Exercise, stress management and social support are also very important for reversing heart disease according to Buckley (2017). The following diets have been shown to reverse heart disease. They include: The Dean Ornish Plan, Caldwell B. Esselstyn's Prevent and Reverse Heart Disease, The McDougall Program and Steven Masley's 30 day Heart Tune-up (Buckley, 2017).

\section{The ornish plan}

Ornish's 37 years of research has scientifically proven that the integrative lifestyle changes he recommends can improve chronic conditions such as heart disease, diabetes and prostate cancer (Buckley, 2017). Ornish's program is the first program scientifically proven to "undo" (reverse) heart disease by optimizing these four areas: Diet, exercise/fitness, stress reduction and emotional support (Buckley, 2017). The clinical results obtained by the Ornish Program were based on the entire package, which involves not only a plant based diet but also physical exercise, stress management techniques including meditation and yoga, and psychological support group sessions (Buckley, 2017).

Ornish's Nutrition Guidelines for Reversing Heart Disease according to Buckley, (2017) are: a. Fat: calories from fat should not be more than $10 \%$. This is achieved by not adding any fats, oils, seeds, nuts, avocados, coconut and olives to a mostly plant based diet. The $10 \%$ of calories from fat comes from fat that occurs naturally in grains, vegetables, fruit, beans, legumes and soy foods.

b. Cholesterol: cholesterol should not be more than $10 \mathrm{mg}$ per day. To meet this goal, non-fat dairy products are limited to 2 servings per day. Non-fat dairy products are optional. Soy products can be used instead of dairy products because they are cholesterol free.

c. Animal Products: animal products like meat, poultry, fish and any products made from these foods are eliminated. Nonfat dairy foods (not more than 2 servings daily) and egg whites are included.

d. Calories: calories should be unrestricted unless weight loss is desired. Small frequent meals spread throughout the day help avoid hunger and keep energy levels constant. Portion control is stressed.

e. Sugar: sugar is permitted in moderation. It should not be more than 2 servings daily including non-fat sweets. A serving is equivalent to 1 tablespoon or 12 grams of sugar.

f. Caffeine: All sources of caffeine are eliminated, including regular and decaffeinated coffees and teas, chocolate, cocoa, and regular or decaffeinated dark colas, with the exception of green tea. Individuals with arrhythmia and elevated stress should still avoid any caffeinated beverage. Green tea should be limited to no more than 2 cups per day. Decaffeinated green tea can be consumed.

g. Sodium: salt should be used moderately, unless medically indicated otherwise.

h. Alcohol: alcohol is allowed in small quantity but not encouraged. If consumed, enjoy one serving a day.

i. Soy: one serving per day of a "full-fat" soy food. A full-fat soy food is one that contains greater than 3 grams of fat per serving, with none of the fat coming from added fats or oils.

j. Supplements: a low dose multivitamin and mineral supplement with B-12 (without iron, if not of childbearing age), fish oil and, possibly upon the advice of a physician, calcium supplements. Antioxidant vitamins and folic acid are optional and are based on health history and nutritional intake of these nutrients. Patients take 4 grams daily of fish oil or flax oil to provide the omega-3 fatty acids.

\section{Esselstyn}

Esselstyn's study was updated at 12 years and reviewed beyond 20 years in his book titled Prevent and Reverse Heart Disease, making it one of the longest longitudinal studies of its type (Buckley, 2017). The program is a nutrition based therapy (Strictly plant based diet, no foods of animal origin at all) that has been scientifically proven to reverse heart disease. Coronary angiograms (X-Rays) of the patients show an actual reversal of heart disease (Buckley, 2017). Esselstyn's Nutrition Guidelines for Reversing Heart Disease according to Buckley, (2017) are:

a. No meat, poultry, or fish. 


\section{International Journal of Engineering Applied Sciences and Technology, 2019 \\ Vol. 4, Issue 3, ISSN No. 2455-2143, Pages 391-399 \\ Published Online July 2019 in IJEAST (http://www.ijeast.com)}

b. No dairy products.

c. No consumption of oil of any kind not even a drop.

d. No nuts or avocado.

e. All Vegetables: all vegetables such as green leafy vegetables, root vegetables, vegetables that are red, green, purple, orange, and yellow are permitted on this plan with the exception of avocados. Avocados have a very high fat content. Those that do not have heart disease may eat avocados as long as blood lipids are not elevated.

f. Legumes: All legumes such as beans, peas, and lentils of all kinds are permitted on this plan.

g. Whole Grains: any grain is permitted as long as it is "whole" grain. "Whole" means that it has not been polished or processed to eliminate much of the nutritional value. Breakfast cereals that do not contain added oil and sugar can be eaten. All whole grains and products, such as bread and pasta that are made from them as long as they do not contain added fats are permitted.

h. Fruits: Fruits of all kinds are permitted. It's best to limit fruit consumption to three servings a day. Also, avoid consuming pure fruit juices since there's excessive amounts of sugar that will elevate the triglycerides. Be careful of all desserts for the same reason.

i. Beverages: The following beverages are permitted. Water, seltzer water, oat milk, no fat soy milk, coffee, and tea. Alcohol is okay in moderation.

j. Supplements: one multivitamin that covers all the basic requirements should be taken daily. $1000 \mathrm{mcg}$ (micrograms) of vitamin B12 should be taken daily. People over 50 years should take 1000 milligrams of calcium daily and people over 60 years should take $1200 \mathrm{mg}$ of calcium daily. Those over 50 years should take 1000 IU of Vitamin D daily.

k. Omega Fatty Acids: one tablespoon of flaxseed meal should be consumed each day. It can be sprinkled on cereal.

1. Cholesterol lowering drugs (if necessary). This must be taken under supervision of a physician.

\section{The Mc Dougall program}

The Mc Dougall program has shown to reverse serious illnesses including high blood pressure, heart disease, diabetes and others, all without the use of drugs but include exercise as simple as a daily walk (Buckley, 2017).

The Mc Douguall's Nutrition Guidelines for Reversing Heart Disease according to Buckley, (2017) are:

a. A diet of plant foods, including whole grains and whole grain products (such as pasta, tortillas, and whole grain bread), and a wide assortment of vegetables and fruits.

b. Plenty of spices and usually small amounts of sugar and salt to enhance the flavour of food.

c. The exclusion of animal foods, including red meat, poultry, dairy products, eggs, and fish. d. The exclusion of all oils including olive oil, safflower oil, and corn oil.

\section{Steven Masley}

Steven Masley has a five point plan for preventing and reversing the progression of heart disease, based on his own clinical studies (Buckley, 2017). In his book, "The 30-Day Heart Tune-Up," he details how to effectively boost the heart health in as little as a few weeks (Buckley, 2017).

Masley has come up with a five-point plan that he calls the "Five Fs" for lowering the risk of heart disease: Fiber, Fitness, Body Fat, Fish and fish oil, Food nutrients (Buckley, 2017).

a. Fiber: Getting enough natural fiber in the diet from vegetables, fruit, beans, and nuts is the single best thing that can boost the heart health. "They are awesome and do so many things," he says. "They help lose weight, they suppress appetite, they are good for the blood sugar, blood pressure and they slow aging."

b. Fitness: The standard minimum guideline of 30 minutes of moderate exercise most days of the week.

c. Body fat: A diet rich in fibre and regular exercise helps burn body fat

d. Fish and fish oils: Masley recommends 1,000 milligrams per day of healthy fish oils, from food or supplements. However, actual fish (salmon, trout, sole) is a better option than supplement.

e. Food Nutrients: Masley recommends 2,000 IUs of vitamin D, 400 milligrams of magnesium, and vitamin $\mathrm{K}$ from green leafy vegetables such as broccoli, kale, or spinach a day.

In addition to incorporating the "Five Fs", Masley advises steering clear of trans fats which he refers to as "embalming fluid" and processed foods high in refined carbohydrates, sugar, and flour (Buckley, 2017).

\section{CONCLUSION}

Eating habits and other lifestyle factors play an important role in determining the risk of heart disease and may prevent or even reverse this condition. Nutrition can play a significant role in preventing, controlling and reversing heart disease if a good dietary pattern is systematically followed. What is eaten can help keep the heart beat strong or lead to overweight, high blood pressure, and high blood cholesterol, which are the three key factors that increase the risk of developing heart disease. The only way to maintain a healthy heart is an allencompassing healthful lifestyle that incorporates a varied, low-fat, vegetarian diet, daily physical activity, and stress reduction.

\section{REFERENCE}

[1] American Heart Association (A.H.A) (2002). A Public Health Plan to Prevent Heart Disease and Stroke. U.S. Department of Health and Human Services Centres for 


\section{International Journal of Engineering Applied Sciences and Technology, 2019 \\ Vol. 4, Issue 3, ISSN No. 2455-2143, Pages 391-399 \\ Published Online July 2019 in IJEAST (http://www.ijeast.com)}

Disease Control and Prevention. USA: American Health Association.

[2] American Heart Association (A.H.A). (2008). American Heart Association Statistics Committee and Stroke Statistics Subcommittee: Heart disease and stroke statistics: 2008 update. Circulation, 117: e25-e146.

[3] Andrea, H. (2018). List of Foods to Reduce Sodium \& Cholesterol. Retrieved from: https://healthyeating.sfgate.com/list-foods-reducesodium-cholesterol-4996.html. (Assessed 06/03/19)

[4] Atli, A. (2015). Beef 101: Nutrition Facts and Health Effects. https://www.healthline.com/nutrition/foods/beef. (Assessed: 03/04/2019).

[5] Aune, D., Keum, N., Giovannucci, E., Fadnes, L. T., Boffetta, P., Greenwood, D. C., Tonstad, S., Vatten, L. J., Riboli, E. and Norat, T. (2016). Nut consumption and risk of cardiovascular disease, total cancer, all-cause and cause-specific mortality: a systematic review and doseresponse meta-analysis of prospective studies. BioMed Central Public Health, 14(1): 207.

[6] Bellows, L. and Moore, R. (2013). Heart Health: Managing Heart Disease through Diet- 9.384. https://extension.colostate.edu/topic-areas/nutrition-foodsafety-health/heart-health-managing-heart-diseasethrough-diet-9-384/. (Assessed: 03/08/2019).

[7] Benjamin, W. (2016). file://C:/Users/user1/Desktop/MSc\%20Seminar/5\%20Ty pes \%20of\%20Heart\%20Disease\%20Symptoms, \%20Risk $\%$ 20Factors, \%20Causes\%20\&\%20Stats.htm. (Accessed: 05/18/17).

[8] Better health channel (2012). Heart disease and food. https://www.betterhealth.vic.gov.au/health/conditionsandt reatments/heart-disease-and-food?viewAsPdf=true. Accessed: 05/28/2017).

[9] Bridget, B. K. (2010). Promoting cardiovascular health in the developing world : a critical challenge to achieve global health. Fuster, Board on Global Health; Valentin; Academies. Institute of Medicine of the National, eds. Washington, D.C.: National Academies Press. pp. Chapter 2.

[10] Brouwer, I. A., Wanders, A. J, Katan, M. B. (2010). Effect of animal and industrial trans fatty acids on HDL and LDL cholesterol levels in humans: a quantitative review. PLoS ONE, 5:e9434.

[11] Borneo, R. and Leon, A. E. (2012). Whole grain cereals: Functional components and health benefits. Food and Function, 3(2): 110-119.

[12] Buckley, S. (2017). Heart disease reversal diets. https://www.southdenver.com/wpcontent/uploads/2012/09/Heart-disease-reversal-diets-nopics.pdf. (Accessed: 07/08/2017).
[13] Channi-Tiwar, H. (2018). 10 Magical Benefits Of Onions That Keep the Doctor Away. https://food.ndtv n7.com/health/10-magical-benefits-of-onions-that-keepthe-doctor-away-1430441. (Accessed: 12/03/2019).

[14] Christian, N. (2016). Heart Disease: Definition, Causes, Research. :http://www.medicalnewstoday.com/articles/237191.php. (Accessed: 05/15/2017).

[15] Chuang, S. C., Norat, T., Murphy, N., Olsen, A., Tjønneland, A., Overvad, K., Boutron-Ruault, M. C., Perquier, F., Dartois, L., Kaaks, R., Teucher, B., Bergmann, M. M., Boeing, H., Trichopoulou, A., Lagiou, P., Trichopoulos, D., Grioni, S., Sacerdote, C., Panico, S., Palli, D., Tumino, R., Peeters, P. H., Bueno-de-Mesquita, B., Ros, M. M., Brustad, M., Åsli, L.A., Skeie, G., Quirós, J.R., González, C.A., Sánchez, M.J., Navarro, C., Ardanaz Aicua, E., Dorronsoro, M., Drake, I., Sonestedt, E., Johansson, I., Hallmans, G., Key, T., Crowe, F., Khaw, K. T., Wareham, N., Ferrari, P., Slimani, N., Romieu, I., Gallo, V., Riboli, E. and Vineis, P. (2012). Fiber intake and total and cause-specific mortality in the European Prospective Investigation into Cancer and Nutrition cohort. American Journal of Clinical Nutrition, 96:164-174.

[16] Craig, W. J. (2009). Health effects of vegan diets. American Journal of Clinical Nutrition, 89: 1627S$1633 \mathrm{~S}$.

[17] Craig, W. J. and Mangels, A. R. (2009). American Dietetic Association. Position of the American Dietetic Association: vegetarian diets. Journal of American Dietetic Association, 109: 1266-1282.

[18] Di Angelantonio, E., Sarwar, N., Perry, P., Kaptoge, S., Ray, K. K., Thompson, A., Wood, A. M, Lewington, S., Sattar, N., Packard, C. J., Collins, R., Thompson, S. G. and Danesh, J. (2009). JAMA, 302(18):1993-2000.

[19] D jousse, L., Arnett, D. K., Coon, H., Province, M. A., Moore, L. L. and Ellison, R. C. (2004). Fruit and vegetable consumption and LDL cholesterol: the National Heart, Lung, and Blood Institute Family Heart Study. American Journal of Clinical Nutrition, 79: 213-217.

[20] Esselstyn, C. B. (2007). Prevent and reverse heart disease. Progress in clinical and biological research, 347355.

[21] Esselstyn, C. B. (1999). Updating a 12-year experience with arrest and reversal therapy for coronary heart disease (an overdue requiem for palliative cardiology). American Journal of Cardiology, 84: 339-341.

[22] Healthwise (2015). Healthy Eating: How to Eat a HeartHealthy Diet. From Healthwise Incorporated. www.CardioSmart.org (Accessed: 13/08/2016).

[23] Hu, F. B. (2003). Plant-based foods and prevention of cardiovascular disease: an overview. American Journal of Clinical Nutrition, 78(suppl):544S-51S. 


\section{International Journal of Engineering Applied Sciences and Technology, 2019 \\ Vol. 4, Issue 3, ISSN No. 2455-2143, Pages 391-399 \\ Published Online July 2019 in IJEAST (http://www.ijeast.com)}

[24] Jakulj, F., Zernicke, K., Bacon, S. L., van Wielingen, L. E., Key, B. L., West, S. G. and Campbell, T. S. (2007). A high-fat meal increases cardiovascular reactivity to psychological stress in healthy young adults. Journal of Nutrition, 137: 935-939.

[25] Kathleen, M. S. and Forrest, W. T. (2009). Heart Healthy Eating: Cholesterol, Fat, Fiber, \& Sodium. Retrieved from: https://pubs.ext.vt.edu/348/348-898/348-898.html. (Assessed 06/03/19).

[26] Lawrence, R., Jeanne, S. and Melinda, S. (2017). HeartHealthy Diet Tips; Eating to Prevent Heart Disease and Improve Cardiovascular Health. https://www.helpguide.org/.(Accessed: 03/08/2019).

[27] Li, T. Y., Brennan, A. M., Wedick, N. M., Mantzoros, C., Rifai, N. and Hu, F. B. (2009). Regular Consumption of Nuts Is Associated with a Lower Risk of Cardiovascular Disease in Women with Type 2 Diabetes. Journal of Nutrition, 139(7): 1333-1338.

[28] Libby, P. (2005). Prevention and treatment of atherosclerosis. In: Braunwald E, Fauci AS, Kasper DL Hauser AL, Longo DL, Jameson JL (eds). Harrison's Principles of Internal Medicine. 16th ed. MacGraw Hill, U.S.A. Pp1430 -1433.

[29] Lichtenstein, A. H, Appel, L. J., Brands, M., Carnethon, M., Daxniels, S., Franch, H. A., Franklin, B., KrisEtherton, P., Harris, W. S., Howard, B., Karanja, N., Lefevre, M., Rudel, L., Sacks, F., Van Horn, L., Winston, M. and Wylie-Rosett, J. (2006). Diet and Lifestyle Recommendations Revision 2006: A Scientific Statement from the American Heart Association Nutrition Committee. Circulation, 114: 82 - 96.

[30] Maresz, K. (2015). Proper Calcium Use: Vitamin K2 as a Promoter of Bone and Cardiovascular Health. Integrative Medicine: Cliniccal Journal (Encinitas), 14(1): 34-9.

[31] Miller, M, Stone, N. J., Ballantyne, C. Bittner, V., Criqui, M. H, Ginsberg, H. N., Goldberg, A. C., Howard, W. J, Jacobson, M. S, Kris-Etherton, P. M, Lennie, T. A, Levi, M., Mazzone, T. and Pennathur, S. (2011). Triglycerides and cardiovascular disease: a scientific statement from the American Heart Association. Circulation, 12320:2292-2333.

[32] Morgan, C. (2015). Heart Disease (Cardiovascular Disease, CVD). http://www.newsmax.com/FastFeatures/heart-diseasetypes/2015/09/21/id/692567/. (Accessed: 05/18/2017).

[33] Mozaffarian, D., Benjamin, E. J., Go, A. S., Arnett, D. K., Blaha, M. J.,, Cushman, M., de Ferranti, S., Després, J. P., Fullerton, H. J., Howard, V. J., Huffman, M. D., Judd, S. E., Kissela, B. M., Lackland, D. T., Lichtman, J. H., Lisabeth, L. D., Liu, S., Mackey, R. H., Matchar, D. B., McGuire, D. K., Mohler, E. R. 3rd, Moy, C. S., Muntner, P., Mussolino, M. E., Nasir, K., Neumar, R. W., Nichol, G., Palaniappan, L., Pandey, D. K., Reeves, M. J.,
Rodriguez, C. J., Sorlie, P. D., Stein, J., Towfighi, A., Turan, T. N., Virani, S. S., Willey, J. Z., Woo, D., Yeh, R. W. and Turner, M. B. (2015). Heart Disease and Stroke Statistics At-a-Glance. https://www.heart.org/idc/groups/ahamahpublic/@wcm/ @ sop/@smd/documents/downloadable/ucm_470704.pdf. (Accessed: 16/ 08/ 2016).

[34] National Heart Foundation of Australia and the Cardiac Society of Australia and New Zealand (2012). Reducing risk in heart disease: an expert guide to clinical practice for secondary prevention of coronary heart disease. Melbourne: National Heart Foundation of Australia, 2012. ISBN 978-1-74345-016-1.

[35] National Heart Lung and Blood institute (N.I.H) (2017). Know the Differences Cardiovascular Disease, Heart Disease, Coronary Heart Disease. https://www.nhlbi.nih.gov/sites/default/files/media/docs/F act_Sheet_Know_Diff_Design.508_pdf.pdf. (Accessed: 06/20/2018).

[36] National Heart, Lung, and Blood Institute (N.I.H) (2003). Keep the Beat Heart Healthy Recipes. National Institutes of Health National Heart, Lung, and Blood Institute, U.S. Department of Health and Human Services. NIH Publication. Pp 1.

[37] Pelkman, C. L., Fishell, V. K., Maddox, D. H., Pearson, T. A., Mauger, D. T. and Kris-Etherton, P. M. (2004). Effects of moderate-fat (from monounsaturated fat) and low-fat weight-loss diets on the serum lipid profile in overweight and obese men and women. The American Journal of Clinical Nutrition, 79(2): 204-212

[38] Physician Committee for Responsible Medicine (2018). Cholesterol and Heart Disease. 5100 Wisconsin Ave., N.W., Suite 400. Washington, DC 20016. Retrieved from: http://pltransform.com/cholesterol-and-heart-disease/. (Accessed: 03/03/2019).

[39] Pretorius, S. and Mpe, D. M. (2016). Nutrition Therapy in Prevention and Treatment of Heart Failure. http://www.hefssa.org/images/uploads/diet_requirements. pdf. (Accessed: 08/23/2016).

[40] Rachael, L. (2018). 15 Incredibly Heart-Healthy Foods. Retrieved from: https://www.healthline.com/nutrition/heart-healthy-foods (Accessed on 08/03/19).

[41] Ried, K. (2016). Garlic Lowers Blood Pressure in Hypertensive Individuals, Regulates Serum Cholesterol, and Stimulates Immunity: An Updated Meta-analysis and Review. Journal of Nutrition, 114-202.

[42] Sandi, B. (2018). Zero Fiber Food. https://healthyeating.sfgate.com/zero-fiber-food7968.html (Accessed: 04/03/2019).

[43] Sandmaier, M. (2005). Your Guide to a Healthy Heart. National Institutes of Health, National Heart, Lung, and 


\section{International Journal of Engineering Applied Sciences and Technology, 2019 \\ Vol. 4, Issue 3, ISSN No. 2455-2143, Pages 391-399 \\ Published Online July 2019 in IJEAST (http://www.ijeast.com)}

Blood Institute, U.S. Department of Health and Human Services. USA: NIH NIH Publication. Pp3-57.

[44] Sauvaget C, Nagano J, Hayashi M, Yamada M (2004) Animal protein, animal fat, and cholesterol intakes and risk of cerebral infarction mortality in the adult health study. Stroke, 35: 1531-1537.

[45] Scherr, R. E. and Zidenberg-Cherr, S. (2016). Nutrition and Health Info Sheet: Cholesterol For Health Professionals. Center for Nutrition in Schools Department of Nutrition University of California, Davis. https://cns.ucdavis.edu/sites/g/files/dgvnsk416/files/inline -files/fact-pro-cholesterol_1.pdf. (Accessed: 02/03/2019).

[46] Smith, K., Qu, W., Ren, X. and Wang, Y. (2015). Does pollution exacerbate obesity risks? A systematic review and meta-analysis. FASEB J, 29(1 Suppl.):736.34

[47] Stephen, S. (2018)a. The Benefits of Garlic (It Does WHAT?). https://heartmdinstitute.com/dietnutrition/benefits-of-garlic-it-does-what/. (Accessed: 03/12/2019).

[48] Stephen, S. (2018)b. The Health Benefits of Turmeric. https://www.drsinatra.com/the-health-benefits-ofturmeric. (Accessed: 03/12/2019).

[49] Valencia, H. and Kristeen, C. (2016). Heart Disease types. http://www.healthline.com/health/heartdisease/types\#over view1. (Accessed: 05/18/2017).

[50] Vikas, K., Rayomand, S. K., Amy, R., Mark, J. C. and Amrita, A. (2014). Dietary nitrate provides sustained blood pressure lowering in hypertensive patients: a randomized, phase 2, double-blind, placebo-controlled study. Hypertension, 65:320-327.

[51] Walker P, Rhubart-Berg P, McKenzie S, Kelling K, Lawrence R. S (2005) Public health implications of meat production and consumption. Public Health Nutrition, 8: 348-356.

[52] World health organisation (W.H.O) (2016). Cardiovascular diseases (CVDs). http://www.who.int/mediacentre/factsheets/fs317/en/. (Accessed: 16/08/ 2016).

[53] World health organisation (W.H.O) (2017). Cardiovascular diseases; the problem. https://www.who.int/nmh/publications/fact_sheet_cardiov ascular_en.pdf. (Assessed: 03/03/2019). 